\title{
Influence of Coronal Mass Ejections from the Red Dwarf Component on the Accretion Pattern in CVs and LMXBs
}

\author{
Roberto Minarini ${ }^{1,2}$, Grigory Beskin ${ }^{2}$ \\ 1 Dipartimento di Astronomia, Università di Bologna, via Zamboni 33, 40126, \\ Bologna, Italy \\ 2 Special Astrophysical Observatory of the Russian Academy of Sciences, \\ Nizhnij Arkhyz, Karachaevo-Cherkessiya, 357147, Russia
}

\section{Introduction}

Low-mass main sequence stars show a magnetic activity similar to the Sun and as a consequence they lose mass in the form of a variable stellar wind. In the latest spectral types (red dwarfs) the activity and the mass loss rate appear to increase by a large factor of $\sim 10^{3}$ with respect to the solar case, reaching $\dot{M} \sim 2 \cdot 10^{-11} \mathrm{M}_{\odot} / \mathrm{yr}$ (Badalyan \& Livshits 1992, Katsova 1993). The same happens for coronal mass ejections (CMEs), which are the most relevant transient events of mass loss in these objects. In the Sun, these appear as bubbles of coronal material, with dimensions of some fraction of the solar surface, mass $M \simeq 2 \cdot 10^{14}-2 \cdot 10^{16} \mathrm{~g}$ and ejection velocity $v \simeq 3 \cdot 10^{7}-2 \cdot 10^{8} \mathrm{~cm} / \mathrm{s}$, with an instantaneous mass loss rate $\dot{M} \sim 10^{-13}-10^{-11} \mathrm{M}_{\odot} /$ yr (Wagner 1984). In red dwarfs, as recently observed, the ejection velocities are higher, up to $v \simeq 3 \cdot 10^{8} \mathrm{~cm} / \mathrm{s}$ and the mass loss rate can reach the value $\dot{M} \simeq 10^{-8} \mathrm{M}_{\odot} / \mathrm{yr}$ (Mullan et al. 1989, Houdebine et al. 1990). In both cases, the observations suggest that a bubble expands, once ejected, with a velocity of several hundreds of $\mathrm{km} / \mathrm{s}$.

\section{Possibility of an accretion event}

We asked ourselves what would happen if a bubble, expelled by the red dwarf component of a cataclysmic variable or a low-mass X-ray binary, is accreted by the compact object. The ejection velocity of the bubble (some thousands of $\mathrm{km} / \mathrm{s}$ ) is one order of magnitude larger than the escape velocity from the red dwarf and the orbital velocity of these systems. Thus, if the bubble travels toward the compact object, it is accreted directly. It is possible in this way to take into account a second stream of accretion in these binary systems. The transfer time scale is of the order of some minutes, much smaller than the accretion disk viscous time scale. It means that a transient mass loss event on the red dwarf can rapidly be followed by a transient event of mass accretion by the compact object. 
The bubble can be expelled from the star in any direction. The accretion probability can be approximated by the ratio between the cross section of the bubble itself and the surface of the sphere with radius equal to the distance between the two components. We estimate $P \sim 10^{-2}$. This must be combined with the production rate of CMEs, which is about 1 /day in the Sun and not yet determined but probably larger in red dwarfs.

\section{Estimates of the emitted luminosity}

Taking the typical values for the mass, density and velocity of a bubble and for the parameters of a binary system, we estimated the amount of energy emitted during the accretion process.

First of all, the accretion radius is of the same order of the radius of the bubble, so a relevant fraction of the gas is effectively accreted. We calculated the accretion luminosity by the formula $L=G M \dot{M} / R$, where $\dot{M}$ is the amount of matter which enters the accretion surface per unit time interval. For a $C V$ we get $L \sim 10^{31}-10^{33} \mathrm{erg} / \mathrm{s}$. If this energy is thermalized in the white dwarf photosphere, it is blackbody emission in optical-UV. It will be bremsstrahlung emission in X-rays from the accretion column of a magnetized white dwarf. From a LMXB we get $L \sim 10^{33}-10^{36} \mathrm{erg} / \mathrm{s}$, in X-rays considering bremsstrahlung emission from a neutron star, magnetized or not.

These luminosities are significant and a burst can be observed as a consequence of a CME, also if the system already emits accretion luminosity. The duration will be of some minutes up to some tens of minutes, corresponding to the time a bubble needs to cross the compact object's accretion sphere. We conclude that the described phenomenon can be a cause of some bursting behaviour in CVs and LMXBs.

\section{References}

Badalyan O.G., Livshits M.A., 1992, Sov. Astron. 36, 70

Houdebine E.R., Foing B.H., Rodonò M., 1990, A\&A 238, 249

Katsova M., 1993, in Inside the Stars, IAU Coll. 137, W.W. Weiss \& A. Baglin (eds.), ASP Conf. Ser. Vol. 40, p. 654

Mullan D.J., Sion E.M., Bruhweiler F.C., Carpenter K.G., 1989, ApJ 339, L33

Wagner W.J., 1984, ARA\&A 22, 267 